\title{
Estudio comparativo entre cesárea tipo Misgav Ladach y cesárea tipo Kerr
}

\author{
Misgav Ladach versus Kerr cesarean section: Comparative study
}

\author{
Walter Ventura \\ Médico Ginecólogo. Instituto Nacional Materno Perinatal. Lima, Perú.
}

\begin{abstract}
Resumen
Introducción: La técnica de cesárea conocida como Misgav Ladach es una técnica minimalista y está siendo usada cada vez más en diferentes partes del mundo. Objetivos: Determinar los beneficios de la técnica Misgav Ladach, comparados con los de la cesárea clásica de Kerr. Diseño: Estudio retrospectivo, comparativo. Lugar: Hospital II EsSalud, Huamanga, Ayacucho. Participantes: Gestantes a término con feto único vivo, con indicación de cesárea. Intervenciones: Cesárea. Principales medidas de resultados: Tiempo operatorio, tiempo de extracción fetal, sangrado operatorio, uso de analgésico en el postoperatorio. Resultados: Los tiempos operatorios promedio fueron 25 vs. 38 minutos, según el empleo de la técnica Misgav-Ladach y Kerr, respectivamente. Los tiempos de extracción fetal fueron 79 vs. 139 segundos, con diferencia significativa. La media de hemoglobina fue de 1,2 $\mathrm{g} / \mathrm{dL}$ en el grupo de casos y 1,47 g/dL en el grupo control, mostrando diferencia significativa. El grupo Misgav-Ladach requirió menor analgesia postoperatoria. No se alcanzó diferencia significativa en la morbilidad febril y en la necesidad de antibiótico terapia poscirugía. Conclusiones: La cesárea MisgavLadach parece mostrar mejores beneficios con respecto a la cesárea clásica, lo cual debe ser confirmado mediante estudios prospectivos aleatorios.

Palabras clave: Cesárea; Kerr, Monro; Ladach, Misgav; estudio comparativo.
\end{abstract}

\begin{abstract}
Introduction: There are reports from several countries on good outcomes with Misgav Ladach's cesarean section technique use. Objetives: To determine results obtained with Misgav Ladach's cesarean technique and to compare them with the traditional Kerr's cesarean section. Design: Retrospective, comparative study. Setting: Hospital II, EsSalud, Huamanga, Ayacucho. Participants: Full term pregnant women with a single fetus and medical indication for cesarean section. Interventions: Cesarean section. Main outcome measures: Surgical time, time to fetal birth, blood loss, use of post operative analgesia. Results: Mean surgical time was 25 and 38 minutes with respectively Misgav Ladach's and Kerr's techniques. Time to fetal birth was 79 seconds in cases vs. 139 seconds in the control group with significant difference. Mean hemoglobin was $1,2 \mathrm{~g} / \mathrm{dL}$ significantly less in cases than 1,47 in the control group. Furthermore, the cases group needed less analgesia than the control group. Fever and need of antibiotics following surgery did not show significant differences. Conclusions: Misgav Ladach's cesarean section seems to be more beneficial than traditional cesarean section, to be confirmed in future studies.

Key words: Cesarean section; Kerr, Monro; Ladach, Misgav; comparative study.
\end{abstract}

\section{INTRODUCCIÓN}

La cesárea es uno de los procedimientos quirúrgicos más practicados en el mundo. Varios historiadores han observado que ya era conocida en el año $715 \mathrm{aC}$. En aquel tiempo, la ley romana lex cesaria preconizaba su uso como una manera de sacar al bebé del vientre de la madre, cuando esta acababa de morir, a fin de enterrarlo separadamente y solo en raras ocasiones para salvar la vida del bebé. En 1897, Pfannestiel propuso el uso de la incisión transversa suprapúbica con una concavidad craneal ${ }^{(1)}$. En 1926, Monro Kerr introdujo la incisión uterina en el segmento inferior de forma transversa ${ }^{(2)}$.

La forma clásica de la cesárea lleva el nombre de cesárea tipo Kerr o Pfannestiel y ha sufrido pocas variaciones al momento actual. Sin embargo, existen varias formas de abordar el abdomen, como lo demostró Joel Cohen, en 1976, con sus investigaciones presentadas en su libro acerca de una nueva forma de apertura del abdomen para realizar histerectomías, el cual fue utilizado posteriormente para las prácticas de cesárea ${ }^{(3)}$.

En 1995, nuestro compatriota Marco A. Pelosi II, en EEUU, informa de un novedoso método para realizar la cesárea, simplificando y omitiendo varios de los pasos que tradicionalmente eran practicados y logrando mejores resultados en cuanto a recuperación y tiempos operatorios ${ }^{(4,5)}$.

El doctor Michael Stark empezó un novedoso método de cesárea, en el año 1983, y comunicó sus resultados en el año 1994, en Montreal, al cual le llamó técnica Misgav-Ladach, que es el nombre del Hospital de Jerusalén donde se inició la práctica de dicho procedimiento ${ }^{(6-9)}$.

En el método de Kerr, el cuerpo es percibido como algo estático y la incisión es hecha disrupcionando toda la anatomía de superficie. Por el contrario, con el método Misgav-Ladach se respeta las estructuras anatómicas y se sigue los principios del minimalismo quirúrgico, resultando aparentemente en un procedimiento más 'fisiológico' que el procedimiento clásico ${ }^{(10)}$.

Actualmente, el método MisgavLadach está siendo probado en ensayos controlados, seleccionados al azar, en varios centros de Suecia, Italia y Kenya (11). Ya se han efectuado estudios comparativos, no al azar, en Israel y Suiza. En América Latina, hay tres trabajos comparativos con esta técnica y en Brasil se está colectando una serie grande de casos, que pronto será publicada. En nuestro país, no se tiene aún comunicaciones con el uso del método.

Las ventajas del método de Misgav Ladach han sido demostradas en diversas publicaciones, y estas incluyen: recuperación postoperatoria rápida, menos reacción febril, menos necesidad 
de administrar antibióticos, el retorno rápido de la función intestinal, menos adherencias peritoneales y menos cicatrización en las capas abdominales (12-15). Se lleva a cabo utilizando solo tres suturas (un catgut crómico 1-0, un vicryl $1-0$ y un catgut crómico 3-0 o nylon 3-0). Todas estas ventajas devienen en un menor costo para las instituciones donde se las practica, lo cual es de resaltar en países en vías de desarrollo, como el nuestro. Asimismo, es una técnica fácil de aprender y reproducir, permitiendo una extracción tan rápida del feto, como las incisiones verticales practicadas tradicionalmente en situaciones de emergencia.

La colaboración Cochrane se refiere a esta técnica como la cesárea del nuevo milenio o la cesárea basada en la mejor evidencia disponible. Desde el año 2004, se encuentra un video de la novedosa técnica en la página web del Grupo de Salud Reproductiva de la Colaboración Cochrane ${ }^{(16)}$.

El objetivo del presente trabajo fue determinar los beneficios de la cesárea según técnica de Misgav Ladach comparados con la técnica convencional descrita por Kerr, en términos de tiempo operatorio, dolor postoperatorio, sangrado intraoperatorio y morbilidad febril postoperatoria.

\section{MÉTODOS}

Se diseñó un estudio de investigación retrospectivo, comparativo, de dos series de casos: serie uno, la cesárea Misgav-Ladach, y serie dos, la cesárea clásica. Se incluyó las gestantes que durante dicho período fueron sometidas a cesárea por el autor, sea de emergencia o de forma electiva, en el Hospital II de EsSalud, de Huamanga, en la ciudad de Ayacucho, desde julio de 2006 hasta julio de 2007. Ambos grupos estuvieron conformados por 71 gestantes. La variable independiente fue el tipo de cesárea y las variables dependientes fueron: 1) tiempo operatorio (tomado en minutos del reporte de anestesiología); 2) tiempo de extracción fetal (tiempo transcurrido desde el inicio de la operación hasta la extracción total del bebé, el cual se encuentra registrada en reporte operatorio); 3) sangrado operatorio (calculado indirectamente según la diferencia de hemoglobina entre el preoperatorio y el postoperatorio de rutina, que se toma dentro de las 24 horas en el hospital); 4) uso de analgesia en el postoperatorio (según la necesidad de administrar analgesia endovenosa luego de 24 horas poscirugía, registrado en la historia clínica; la medicación estándar pasadas las 24 horas fue paracetamol $1 \mathrm{~g} \mathrm{VO} \mathrm{c/8} \mathrm{ho-}$ ras); y 5) estancia hospitalaria, desde la cirugía hasta el alta médica; no se consideró las estancias prolongadas, por razones administrativas. Consideramos variables intervinientes la edad y peso materno, edad gestacional, paridad, cesárea previa. Se incluyó las gestantes con indicación de cesárea. Se excluyó las gestantes con cesárea corporal -porque las dos practicadas en ese período tenían incisión mediana-, aquellas gestantes con peso mayor de $90 \mathrm{~kg}$-por el riesgo de macrosomía fetal-, historias clínicas con datos incompletos y las pacientes en quienes se utilizó anestesia general, porque las 3 registradas fueron operadas con cesárea Misgav Ladach.

Para el análisis bivariado, se utilizó t student, prueba de Mann Whitney y chi2, según correspondiera. En cada una de las mediciones se consideró un error alfa de 0,05 y un intervalo de confianza de 95\%. Todo el análisis se llevó a cabo con ayuda del programa STATA 9,0.

En la tabla 1 se describe las características comparativas de las cesáreas tipos Misgav Ladach y Kerr ${ }^{(8,15,17)}$.

\section{RESULTADOS}

En la tabla 2 se compara los grupos según sus características generales -edad, peso materno, edad gestacional, nulípara y cesárea previa-, sin diferencias significativas entre ellos. En la tabla 3 se muestra las principales indicaciones de cesárea, siendo las distocias de parto y la incompatibilidad cefalopélvica las más frecuentes para la realización de ambas técnicas de cesárea.
Se obtuvo menor tiempo operatorio con la técnica de Misgav-Ladach, siendo el promedio de 25 minutos, con una diferencia estadísticamente significativa $(p<0,05)$. Asimismo, el tiempo de extracción fetal fue menor, siendo la media de 79 segundos, muy por debajo del grupo control (cesárea Kerr), que fue de 139 segundos, con diferencia significativa $(p<0,05)$ (tabla 4$)$.

Se observó además que los pacientes operados con la técnica de MisgavLadach requirieron menor analgesia endovenosa en el posparto mediato, con diferencia también significativa $(p<0,05)$ (tabla 4).

La variación promedio de hemoglobina fue $1,2 \mathrm{~g} / \mathrm{dL}$ en el grupo de quienes fueron operados con la técnica de Misgav-Ladach y de 1,47 g/dL en el grupo de quienes fueron operados con la técnica Kerr, mostrando diferencia significativa $(p<0,05)$, lo cual indica indirectamente menor sangrado en el intraoperatorio (tabla 4).

No existieron diferencias significativas entre ambas técnicas, en cuanto a la morbilidad febril y en la necesidad de antibiótico después de la cirugía (tabla 4).

\section{DISCUSIÓN}

La técnica de Misgav-Ladach, iniciada por el profesor Stark, desde 1983, recién se empieza a conocer después de los Congresos Mundiales de Ginecología y Obstetricia de la FIGO, en Montreal 1994 y en Copenhague 1997, donde se presentó las primeras comunicaciones de dicha técnica. Está ganando gran aceptación por varios centros de práctica obstétrica moderna, no solo en países en vías de desarrollo sino también en países desarrollados ${ }^{(18,19)}$.

La técnica es muy confundida con la técnica del Dr. Marco A. Pelosi II, que introdujo un novedoso método de cesárea también simplificada, en el año 1995. Sin embargo, son técnicas diferentes y no deben ser confundidas ${ }^{(4)}$.

La técnica de Misgav-Ladach ofrece una serie de ventajas, que combinadas 
Tabla 1. Características y comparación entre los pasos de las técnicas de cesárea Misgav Ladach y Kerr.

\begin{tabular}{|c|c|}
\hline Técnica Misgav Ladach & Técnica clásica de Kerr \\
\hline $\begin{array}{l}\text { - } \quad \text { Se inicia con una incisión transversa superficial } \\
\text { de piel, a } 3 \mathrm{~cm} \text { debajo de la línea imaginaria } \\
\text { que une ambas espinas ilíacas anterosuperior } \\
\text { y que mide aproximadamente } 15 \text { a } 16 \mathrm{~cm} \text {. } \\
\text { Aquí hicimos una modificación, al practicar la } \\
\text { incisión a } 203 \mathrm{~cm} \text { por encima de la sínfisis púbica. }\end{array}$ & $\begin{array}{l}\text { - La técnica se inicia con una incisión transversa } \\
\text { superficial, a } 102 \mathrm{~cm} \text { por encima de la sínfisis } \\
\text { púbica, con una ligera concavidad craneal. } \\
\text { La incisión mide en promedio } 14 \mathrm{~cm} \text {. }\end{array}$ \\
\hline $\begin{array}{l}\text { - Incisión del tejido subcutáneo en una extensión } \\
\text { no mayor de } 3 \text { a } 5 \mathrm{~cm} \text {, hasta llegar a la } \\
\text { aponeurosis que cubre los músculos rectos. }\end{array}$ & $\begin{array}{l}\text { - Incisión del tejido subcutáneo y divulsión roma } \\
\text { del tejido graso, hasta visualizar toda la } \\
\text { aponeurosis en la misma extensión que la } \\
\text { incisión de piel. }\end{array}$ \\
\hline $\begin{array}{l}\text { - Incisión en la misma dirección de la } \\
\text { aponeurosis del estuche anterior de } \\
\text { los rectos, en una extensión de } 3 \text { a } 5 \mathrm{~cm} \text {, } \\
\text { desprendiéndola del rafe medio. }\end{array}$ & $\begin{array}{l}\text { - Incisión en la misma dirección de la } \\
\text { aponeurosis anterior del estuche de los rectos, } \\
\text { hasta visualizar los músculos en toda su } \\
\text { extensión, y luego extensión del corte con tijera. }\end{array}$ \\
\hline $\begin{array}{l}\text { - Divulsión y tracción de la aponeurosis junto } \\
\text { con los músculos rectos, en forma paralela a } \\
\text { la incisión de la piel. }\end{array}$ & $\begin{array}{l}\text { - Divulsión roma de los músculos en dirección } \\
\text { cráneo caudal y luego en dirección paralela } \\
\text { a la incisión. }\end{array}$ \\
\hline - $\quad$ Apertura del peritoneo parietal con los dedos. & - $\quad$ Apertura del peritoneo parietal con tijera. \\
\hline $\begin{array}{l}\text { - Incisión del segmento uterino a } 1 \mathrm{~cm} \text { por encima } \\
\text { del repliegue vesicouterino. No se disecciona } \\
\text { el peritoneo visceral. }\end{array}$ & $\begin{array}{l}\text { - Incisión del peritoneo visceral, rechazando la } \\
\text { vejiga e incisión del segmento uterino en el } \\
\text { área que ocupaba el peritoneo visceral. }\end{array}$ \\
\hline $\begin{array}{l}\text { - Extracción fetal en cefálica o podálica, según } \\
\text { presentación. }\end{array}$ & $\begin{array}{l}\text { - Extracción fetal en cefálica o en podálico, } \\
\text { según presentación. }\end{array}$ \\
\hline - Extracción de la placenta por tracción continua. & - Extracción de la placenta por tracción continua. \\
\hline $\begin{array}{l}\text { Escobillonaje de la cavidad uterina con gasa, } \\
\text { por dos veces. }\end{array}$ & $\begin{array}{l}\text { Escobillonaje de la cavidad uterina con gasa, } \\
\text { por dos veces. }\end{array}$ \\
\hline $\begin{array}{l}\text { - Cierre de la pared uterina sin exteriorización, } \\
\text { en un plano con sutura continua y cruzada, } \\
\text { empleando catgut crómico } 1 .\end{array}$ & $\begin{array}{l}\text { - Cierre de la pared uterina, exteriorizándola } \\
\text { de la cavidad, en un plano con sutura continua } \\
\text { y cruzada, empleando catgut crómico } 1 .\end{array}$ \\
\hline $\begin{array}{l}\text { - } \quad \text { Cierre de aponeurosis con sutura continua, } \\
\text { sin cruzar, empleando vicryl } 1-0 .\end{array}$ & $\begin{array}{l}\text { - Cierre del peritoneo visceral y parietal con } \\
\text { catgut crómico 2-0. Cierre de la aponeurosis } \\
\text { con sutura continua cruzada, con vicryl 1-0. }\end{array}$ \\
\hline $\begin{array}{l}\text { - } \quad \text { Afronte del tejido celular subcutáneo, si la } \\
\text { profundidad es mayor de } 2 \mathrm{~cm} \text {, empleando } \\
\text { catgut simple 2-0. }\end{array}$ & $\begin{array}{l}\text { - } \quad \text { Afronte del tejido celular subcutáneo } \\
\text { empleando catgut simple 2-0. }\end{array}$ \\
\hline $\begin{array}{l}\text { - Cierre de piel con sutura subcuticular, } \\
\text { empleando nylon 3-0. }\end{array}$ & $\begin{array}{l}\text { - Cierre de piel con sutura subcuticular, } \\
\text { empleando nylon 3-0. }\end{array}$ \\
\hline
\end{tabular}

culminan en menor tiempo quirúrgico, menor sangrado, recuperación más rápida, menor requerimiento de analgésicos, de antibióticos y menor estancia hospitalaria. Esta técnica es la consecuencia lógica de la introducción de nuevas estrategias quirúrgicas encaminadas a producir menor daño en los tejidos y mínima manipulación. La técnica de Misgav-Ladach se inicia con una incisión Joel Cohen, que es la base principal de esta técnica, y que tracciona los vasos sanguíneos y las fibras nerviosas que tienen cierta elasticidad, desplazándolas hacia un lado, evitando desgarros y conservando la integridad anatómica de los tejidos. Como resultado, existe mínimo sangrado y menos traumatismo para los tejidos, lo que influye directamente sobre la recuperación de la paciente. Con esta técnica no se sutura el peritoneo, en capa alguna, y se plantea que la reformación del tejido peritoneal se completa dentro de las primeras 48 a 72 horas posterior al daño (20-22). 
Tabla 2. Características generales de la población estudiada.

\begin{tabular}{|c|c|c|c|c|c|}
\hline & \multicolumn{2}{|c|}{ Misgav Ladach } & \multicolumn{2}{|c|}{ Kerr } & \multirow{2}{*}{$\begin{array}{l}\text { Significancia } \\
\text { (IC 95\%) }\end{array}$} \\
\hline & $n=71$ & Rango $0 \%$ & $n=71$ & Rango $0 \%$ & \\
\hline $\begin{array}{l}\text { Edad promedio } \\
\text { (años) }\end{array}$ & 32,7 & $(17-46)$ & 31,5 & $(18$ - 44) & $\begin{array}{c}(-0,76,2,98) \\
\text { NS }\end{array}$ \\
\hline $\begin{array}{l}\text { Peso materno } \\
\text { promedio }(\mathrm{kg})\end{array}$ & 65,8 & $(51-80)$ & 66,7 & $(55-84)$ & $\begin{array}{c}(-2,90,0,80) \\
\text { NS }\end{array}$ \\
\hline $\begin{array}{l}\text { Edad gestacional } \\
\text { promedio (semana) }\end{array}$ & 39,1 & $(34-42)$ & 39,5 & $(35-42)$ & $\begin{array}{c}(-0,84,0,11) \\
\text { NS }\end{array}$ \\
\hline Nulípara & 40 & $56,3 \%$ & 37 & $52,1 \%$ & NS \\
\hline Cesárea previa & 9 & $12,7 \%$ & 13 & $18,3 \%$ & NS \\
\hline
\end{tabular}

$\mathrm{NS}=$ no significativo.

El presente estudio demuestra los claros beneficios de la técnica MisgavLadach, en términos de menor tiempo operatorio, menor tiempo de extracción fetal, lo cual es muy útil en situaciones de urgencia o cuando se opera con anestesia general. No incluimos en nuestro estudio pacientes con anestesia general, por no tener los controles necesarios; pero, todas las cesáreas efectuadas durante ese período con anestesia general fueron con la técnica de Misgav Ladach y con tiempos realmente muy cortos.

Hubo mayor necesidad de analgesia endovenosa, en el posparto mediato del grupo con técnica de Kerr, con diferencia estadísticamente significativa, lo cual nos reflejaría mayor dolor postoperatorio. El dolor postoperatorio lo valoramos cualitativamente y en forma indirecta, según requirió o no analgesia endovenosa (metamizol 1,5 a 2 g endovenoso) pasadas las 24 horas, puesto que las primeras horas todas recibían analgesia endovenosa horaria y luego de las cuales pasaban a vía oral.

Asimismo, el grupo con técnica Misgav Ladach mostró menor sangrado intraoperatorio, lo cual lo valoramos según la metodología de otros estudios, con la variación de hemoglobina previo a la cirugía y en un control, luego de 24 horas de la misma ${ }^{(5,23)}$.

No encontramos diferencia significativa en morbilidad febril postoperatoria; tampoco en tiempo de estancia hospitalaria y el uso de antibióticos. Asimismo, todas nuestras pacientes recibieron dosis profiláctica de cefazolina, 2 gramos endovenosos, en el intraoperatorio, según nuestro protocolo.

Creemos que nuestro estudio realizado en una provincia del país tiene gran valor, por ser uno de los pocos publicados, a pesar de las características del diseño. Sin embargo, los resultados son igual de prometedores; así por ejemplo, Monteverde y col, en una serie similar a la nuestra en un hospital universitario de Venezuela, encontraron que el grupo sometido a cesárea Misgav-Ladach $(n=40)$ presentó menor tiempo operatorio (media de 23 minutos) comparado con el grupo control, y un menor tiempo de extracción fetal, con una media de 2,75 minutos ${ }^{(24)}$.

Otros estudios también muestran resultados similares a los nuestros, aunque con mejores tiempos operatorios, en promedio 20 minutos o aún menos, debido a la mayor experiencia con este tipo de técnica ${ }^{(25-27)}$. Asimismo, se logra demostrar en series más grandes el menor tiempo de estancia hospitalaria y menor tasa de morbilidad postoperatoria ${ }^{(28-31)}$.

El presente estudio es retrospectivo y compara dos series de casos operadas por

Tabla 3. Indicaciones de la cesárea.

\begin{tabular}{lccccc}
\hline & \multicolumn{2}{c}{ Misgav Ladach } & & \multicolumn{2}{c}{ Kerr } \\
\cline { 2 - 3 } \cline { 5 - 6 } & $\mathrm{n}=71$ & $\%$ & & $\mathrm{n}=71$ & $\%$ \\
\hline Distocia de parto & 22 & 31 & & 19 & 26,7 \\
Desproporción cefalopélvica & 15 & 21 & 16 & 22,5 \\
Presentación podálica & 10 & 14 & 13 & 18,3 \\
Insuficiencia placentaria & 8 & 11 & 5 & 7 \\
Cesárea previa & 5 & 7 & 9 & 12,8 \\
Otro & 11 & 16 & 9 & 12,8 \\
\hline
\end{tabular}


Tabla 4. Resultados en ambos grupos.

\begin{tabular}{|c|c|c|c|c|c|}
\hline & \multicolumn{2}{|c|}{ Misgav Ladach } & \multicolumn{2}{|c|}{ Kerr } & \multirow{2}{*}{$\begin{array}{l}\text { Significancia } \\
\text { (IC 95\%) }\end{array}$} \\
\hline & $n=71$ & Rango o \% & $n=71$ & Rango o \% & \\
\hline $\begin{array}{l}\text { Tiempo operatorio } \\
\text { promedio (minutos) * }\end{array}$ & 25 & $(16-50)$ & 38 & $(28-65)$ & $\begin{array}{c}(-15,94,-11,18) \\
p<0,0001\end{array}$ \\
\hline $\begin{array}{l}\text { Tiempo de extracción } \\
\text { fetal promedio (segundos) }\end{array}$ & 79 & $(33-142)$ & 139 & $(61-300)$ & $\begin{array}{c}(-72,93,-47,15) \\
p<0,001\end{array}$ \\
\hline $\begin{array}{l}\text { Morbilidad febril } \\
\text { No } \\
\text { Sí }\end{array}$ & $\begin{array}{c}68 \\
3\end{array}$ & $\begin{array}{l}95,8 \% \\
4,2 \%\end{array}$ & $\begin{array}{c}64 \\
7\end{array}$ & $\begin{array}{c}90,1 \% \\
9,9 \%\end{array}$ & $\begin{array}{c}\text { NS } \\
p=0,189\end{array}$ \\
\hline $\begin{array}{l}\text { Antibiótico postoperatorio } \\
\text { No } \\
\text { Sí }\end{array}$ & $\begin{array}{c}65 \\
6\end{array}$ & $\begin{array}{l}91,6 \% \\
8,4 \%\end{array}$ & $\begin{array}{l}59 \\
12\end{array}$ & $\begin{array}{l}83,1 \% \\
16,9 \%\end{array}$ & $\begin{array}{c}\text { NS } \\
p=0,130\end{array}$ \\
\hline $\begin{array}{l}\text { Analgesia endovenosa } \\
\text { mayor de } 24 \text { horas* } \\
\text { No } \\
\text { Sí }\end{array}$ & $\begin{array}{l}57 \\
14\end{array}$ & $\begin{array}{l}80,3 \% \\
19,7 \%\end{array}$ & $\begin{array}{l}46 \\
25\end{array}$ & $\begin{array}{l}64,8 \% \\
25,2 \%\end{array}$ & $p=0,038$ \\
\hline $\begin{array}{l}\text { Variación de la hemoglobina } \\
\text { en } 24 \text { horas }^{*}\end{array}$ & 1,20 & $(0,40-2,3)$ & 1,47 & $(0,6-2,8)$ & $\begin{array}{c}(-0,43,-0,12) \\
p<0,001\end{array}$ \\
\hline $\begin{array}{l}\text { Tiempo de estancia } \\
\text { hospitalaria (días) }\end{array}$ & 3,5 & & 3,6 & & $\begin{array}{c}\text { NS } \\
p=0,8\end{array}$ \\
\hline
\end{tabular}

* Alcanzaron significancia $p<0,05$. NS = no significativo.

el mismo autor en el mismo periodo de tiempo, por lo que los resultados deben ser interpretados teniendo en cuenta los sesgos metodológicos propios del diseño. Sin embargo, puede ser considerado como parte de nuestras herramientas, por los beneficios también demostrados en otras series en términos de menor tiempo operatorio, menor tiempo de extracción fetal, menor sangrado intraoperatorio y menor necesidad de analgesia endovenosa en el postoperatorio mediato.

\section{REFERENCIAS BIBLIOGRÁFICAS}

1. Pfannestiel J. On the advantages of a transverse cut of the fascia the symphysis for gynecological laparotomies, and advice on surgical methods and indications. Samml Klin Vortr Gynakol (neue Folge). 1897:68-98.

2. Kerr JMM. The technique of cesarean section, with special reference to the lower uterine segment. Am J Obstet Gynecol. 1926;12:729-34.

3. Joel-Cohen S. Abdominal and vaginal hysterectomy. New techniques based on time and motion studies. London: Heinemann; 1972. p. 170.

4. Pelosi MA, Pelosi MA Il. Simplified cesarean section. Contemp Obstet Gynecol. 1995;40:89-100.
5. Novoa S. Cesárea tipo Pelosi. Ginecol Obstet (Perú). 2002; 48(4):225-9.

6. Popkind DR, Peddle L. Women's Health Today. Perspectives on current research and clinical practice. The proceedings of the XIV World Congress of Gynecology and Obstetrics. Montreal, Canada. September, 1994.

7. Stark M, Chavin Y, Kupfersztain C, Guedj P, Finkel AR. Evaluation of combinations of procedures in caesarean section. Int J Gynaecol Obstet. 1995;48(3):273-6.

8. Holmgren G. Sjöholm L. The Misgav Ladach method for cesarean section: method description. Acta Obstet Gynecol Scand. 1999;78:615-21.

9. Seligman R. El método Misgav Ladach: efecto máximo, daño mínimo. Rev Shalom. 1997;2:142-6.

10. Stark M. Letter to the Editor: Adhesions. World J Surg. 1993;17:419.

11. Beki D, Kos M. Comparative study of the "Misgav Ladach" and traditional Pfannenstiel surgical techniques for cesarean section. Minerva Ginecol. 2007;59(3):231-40.

12. Ansaloni L, Brundisini R. Prospective, randomized, comparative study of Misgav Ladach versus traditional cesarean section at Nazareth Hospital, Kenya. World I Surg. 2001;25(9):1164-72.

13. Bjarklund K, Kimaro M. Introduction of the Misgav Ladach caesarean section at an African tertiary centre: a randomised controlled trial. BJOG. 2000;107(2):209-16.
14. Escribano JJ, Álvarez A. Comparación de costes de la cesárea mediante técnicas de Misgav Ladach y convencional con incisión de Pfannenstiel. Progr Obstet Ginecol. 2004;47:323-9.

15.Xavier P, Ayres-De-Campos D. The modified Misgav-Ladach versus the Pfannenstiel-Kerr technique for cesarean section: a randomized trial. Acta Obstet Gynecol Scand. 2005;84(9):87882.

16. OMS. Biblioteca de Salud Reproductiva [Internet]. CD versión 10. Grupo Colaborativo Cochrane [citado el 12 de agosto de 2009]. Disponible en: http://apps.who.int/rhl/videos/es/index.html

17. Gibbs RS, Karlan BY. Danforth's Obstetrics and Gynecology. Third Edition. Philadelphia: Lippincott Wiliams and Wilkins; 2008. p. 491-503.

18. FatusićZ, Kurjak A. The Misgav Ladach method--a step forward in operative technique in obstetrics. J Perinat Med. 2003;5:395-8.

19. Federici D, Lacelli B. Cesarean section using the Misgav Ladach method. Int J Gynaecol Obstet. 1997;57(3):273-9.

20. Houth JC, Owen I, Davis RO. Transverse uterine incision closure: one versus two layers. Am J Obstet Gynaecol. 1992;167:1108-11.

21. Hull DB, Varma HW. Randomised study of closure of the peritoneum at caesarean delivery. Obstet Gynecol. 1991;77:818-20.

22. Joura E. Letter: Non-closure of peritoneum and adhesions: The repeat cesarean section. Acta Obstet Gynecol Sacand. 2001;80:286. 
23. Wood RM, Simon H. Pelosi-type vs. traditional cesarean delivery. A prospective comparison. J Reprod Med. 1999;44(9):785-95.

24. Monteverde G, Coromot E, Sanchez AJ. E método para cesárea de Misgav Ladach. Nueva alternativa quirúrgica. Indexmedico Journal [Internet]. 2004(10) [citado el 12 de agosto de 2009]. Disponible en: http://www.indexmedico. com/publicaciones/indexmed_journal/edicion 10/ cesarea/monteverde.htm

25. Guerra P, Fernández JM. Técnica simplificada de operación cesárea versus técnica convencional. Análisis clínico comparativo de dos técnicas de operación cesárea. An Med. 2006;51(4):159 63.

26. Oviedo JG, Buen El. Técnica simplificada de operación cesárea. Estudio clínico comparativo con técnica convencional. An Med Asoc Med Hosp ABC. 2002;47(1):24-8.

27. Tamayo JG, Sereno JA. Comparación entre cesárea Misgav-Ladach y cesárea tradicional. Ginecol Obstet Mex. 2008;76(2):75-80.

28. Habek D, Kulas T. The Misgav Ladach method for cesarean section. Acta Med Croatica. 2007;61(2):153-60.

29. Moreira P, Moreau J. Comparison of two cesarean techniques: classic versus Misgav Ladach cesarean. J Gynecol Obstet Biol Reprod. 2002;31(6):572-6.

30.Studzinski Z. The Misgav-Ladach method for cesarean section compared to the Pfannenstiel technique. Ginekolog Pol. 2002;73(8):672-6.
31.Ziencowicz Z, Suchocki S. Cesarean section by the Misgav Ladach with the abdominal opening surgery by the Joel Cohen method. Ginekol Pol. 2000;71(4):284-7.

Manuscrito recibido el 22 de julio de 2009 y aceptado para publicación el 4 de setiembre de 2009.

\section{Correspondencia:}

Dr. Walter Ricardo Ventura Laveriano

Correo-e: walterichard@hotmail.com 